\title{
Tissue-specific differences in sterol regulatory element binding protein and lipogenic enzyme gene expression in response to dietary cholesterol and fat
}

\author{
S. Kugler, E. Baldry, T. Vallim and A. Salter \\ Division of Nutritional Sciences, School of Biosciences, University of Nottingham, Sutton Bonington Campus, \\ Loughborough, Leics, LE12 5RD, UK
}

Dietary lipids are thought to regulate the expression of lipogenic enzymes, primarily through modulation of the activity of sterol regulatory element binding proteins (SREBP) ${ }^{(1)}$. The present study investigated the effects of dietary cholesterol and fat on the tissue-specific expression of SREBP and of the lipogenic enzymes acetyl-CoA carboxylase-1 (ACC) and fatty acid synthase (FAS).

Thirty-two male hamsters were divided into four groups of eight animals that were fed: low-cholesterol low-fat chow (LC/LF); chow supplemented with $0.2 \%$ cholesterol (w/w; HC/LF); chow supplemented with fat (HF/LC); chow supplemented with cholesterol and fat (HF/HC). High-fat diets were supplemented with $15 \%$ fat $(\mathrm{w} / \mathrm{w})$ blended to produce a fatty acid composition similar to that of a typical 'Western' diet. After 4 weeks animals were killed and mRNA isolated from perirenal adipose tissue and liver. cDNA was prepared and SREBP isoform (1a, 1c and 2), ACC and FAS mRNA were quantified by quantitative real-time PCR. Tissue mRNA concentrations were normalized to TATA-box binding protein mRNA, which was unaffected by diet. Data was analysed by two-way ANOVA with dietary fat and cholesterol as independent factors.
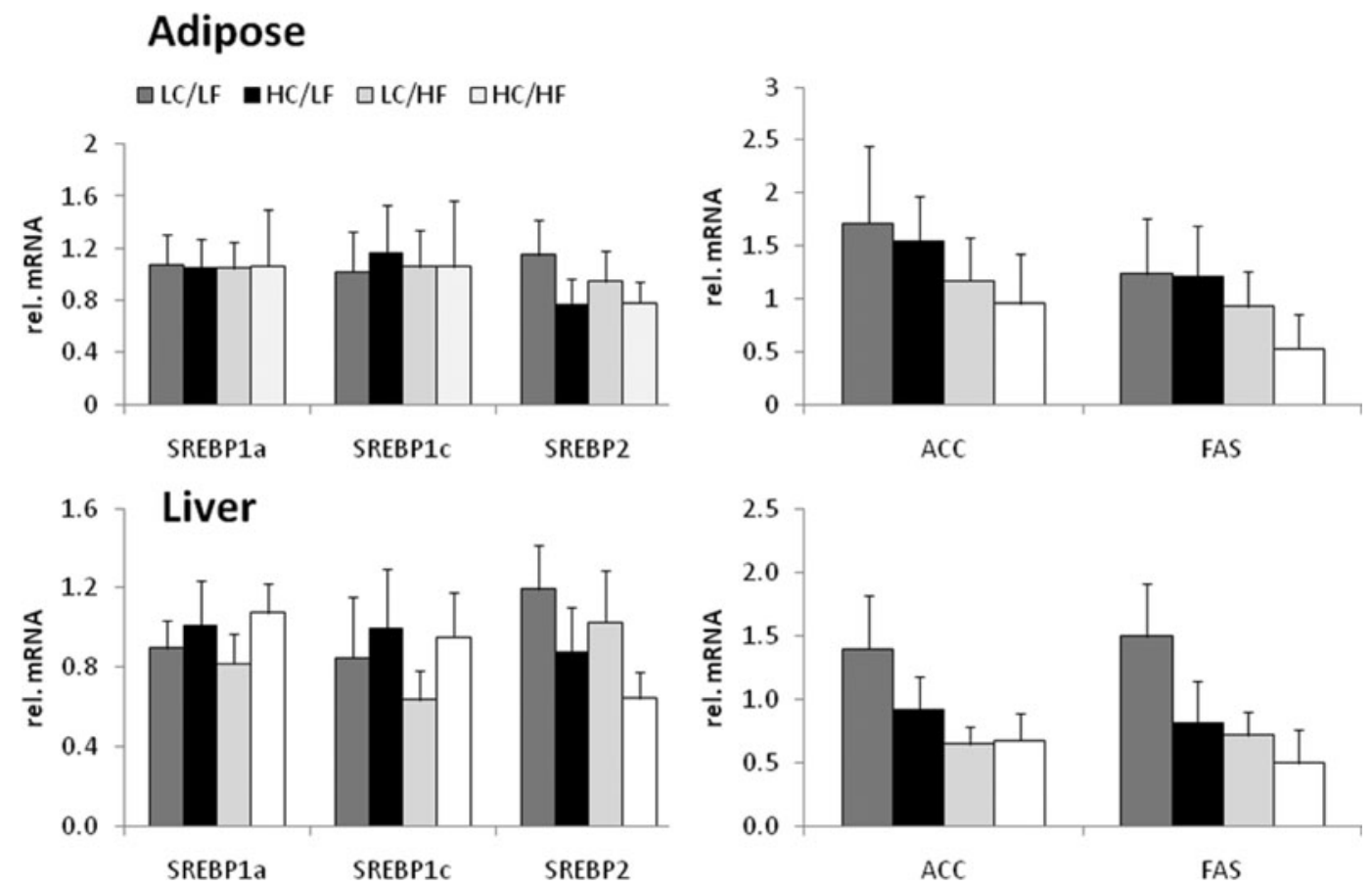

Neither dietary cholesterol nor fat had any significant effect on mRNA concentrations for any of the SREBP isoforms in adipose tissue. By contrast dietary cholesterol significantly raised hepatic SREBP1a $(P=0.006)$ and 1c $(P=0.019)$ mRNA concentrations but decreased SREBP2 $(P<0.001)$. There was no significant interaction between cholesterol and fat in their effect on hepatic SREBP mRNA. In adipose tissue both ACC $(P=0.006)$ and FAS $(P=0.003)$ mRNA concentrations were decrease by dietary fat but unaffected by cholesterol. Both cholesterol and fat reduced hepatic expression of these lipogenic enzymes with significant interactive effects on both ACC $(P=0.019)$ and FAS $(P=0.02)$ mRNA. In liver, but not adipose tissue, there was a highly significant correlation between SREBP2 mRNA concentration and that of both ACC $(r 0.65, P<0.001)$ and FAS $(r 0.68, P<0.001)$.

It is concluded that dietary cholesterol and fat may regulate hepatic lipogenic gene expression through modulating the expression of SREBP2. However, in adipose tissue the reduction in ACC and FAS gene expression must be mediated by other factors.

This study was supported by a British Heart Foundation PhD studentship to T. V. and a BBSRC Summer Studentship to E. B.

1. Raghow R, Yellaturu C, Deng X et al. (2008) Trends Endocrinol Metab 19, 65-73. 